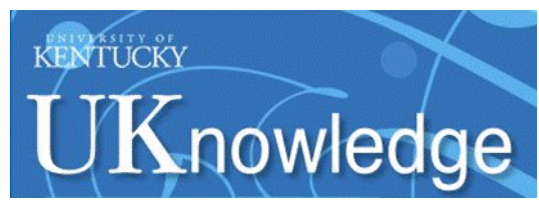

University of Kentucky

UKnowledge

$4-1-2018$

\title{
Dental Students' Skills Assessments: Comparisons of Daily Clinical Grades and Clinical and Laboratory Assessments
}

\author{
Elizangela Bertoli \\ University of Kentucky, ebert2@email.uky.edu \\ Kristy Pepper Lawson \\ University of Kentucky, kristy.pepper@uky.edu \\ Susan S. Bishop \\ University of Kentucky, susan.s.bishop@uky.edu
}

Follow this and additional works at: https://uknowledge.uky.edu/ohp_facpub

Part of the Dental Public Health and Education Commons, and the Educational Assessment, Evaluation, and Research Commons

Right click to open a feedback form in a new tab to let us know how this document benefits you.

\section{Repository Citation}

Bertoli, Elizangela; Lawson, Kristy Pepper; and Bishop, Susan S., "Dental Students' Skills Assessments: Comparisons of Daily Clinical Grades and Clinical and Laboratory Assessments" (2018). Oral Health Practice Faculty Publications. 7.

https://uknowledge.uky.edu/ohp_facpub/7

This Article is brought to you for free and open access by the Oral Health Practice at UKnowledge. It has been accepted for inclusion in Oral Health Practice Faculty Publications by an authorized administrator of UKnowledge. For more information, please contact UKnowledge@lsv.uky.edu. 


\section{Dental Students' Skills Assessments: Comparisons of Daily Clinical Grades and Clinical and Laboratory Assessments}

\section{Digital Object Identifier (DOI)}

https://doi.org/10.21815/JDE.018.045

\section{Notes/Citation Information}

Published in Journal of Dental Education, v. 82, no. 4, p. 417-423.

Reprinted by permission of Journal of Dental Education, Volume 82, 4 (April 2018). Copyright 2018 by the American Dental Education Association. http://www.jdentaled.org 


\title{
Dental Students' Skills Assessments: Comparisons of Daily Clinical Grades and Clinical and Laboratory Assessments
}

\author{
Elizangela Bertoli, DDS, MS; Kristy Pepper Lawson, DMD; Susan S. Bishop, DMD, MHA \\ Abstract: The aim of this study was to compare the daily clinical grades of third-year dental students during routine clinical \\ activities involving direct and indirect operative procedures to clinical and laboratory assessments. The authors compared students' \\ daily clinical grades to graded clinical assessments and compared daily clinical grades to laboratory assessments at one U.S. dental \\ school. A total of 50 third-year students (participation rate: 98\%) participated in this study during the school year of 2014-15. \\ The study analyzed the students' daily clinical grades and graded assessments during regular clinical activities and two laboratory \\ examinations. The results found no statistically significant differences between daily grades and the clinical assessments \\ $(\mathrm{p}=0.2845)$. There were statistically significant differences between daily clinical grades and laboratory assessments $(\mathrm{p}=0.0024)$. \\ This study found higher grades were given for procedures completed and graded in the clinical setting. Further studies are needed \\ to explore the possibility of clinical grades being positively skewed. \\ Dr. Bertoli is Associate Professor, Department of Oral Health Practice, Division of Restorative Dentistry, College of Dentistry, \\ University of Kentucky; Dr. Lawson is Assistant Professor, Department of Oral Health Practice, Division of Comprehensive \\ Care, College of Dentistry, University of Kentucky; and Dr. Bishop is Assistant Professor, Department of Oral Health Practice, \\ Division of Restorative Dentistry, College of Dentistry, University of Kentucky. Direct correspondence to Dr. Elizangela Bertoli, \\ University of Kentucky College of Dentistry, 800 Rose Street, D638 Chandler Medical Center, Lexington, KY 40536-0297; \\ 859-323-4919; ebert2@email.uky.edu.
}

Keywords: dental education, educational assessment, academic performance, patient simulation, clinical education, operative dentistry

Submitted for publication 4/4/17; accepted 10/17/17 doi: 10.21815/JDE.018.045

$\mathrm{L}$ aboratory and clinical settings are essential components in dental education. The two environments combined are important to integrate and implement the various domains of learning: cognitive (thinking), affective (emotion/feeling), and psychomotor (physical/kinesthetic). Traditionally, prior to practice on patients, students are exposed to preclinical didactic classes in which concepts of the procedures are introduced followed by laboratory classes. Laboratory settings, where students are able to perform procedures on a dentoform attached to a simulator, allow for repetition of procedures, facilitating students' hand skills and manual dexterity by developing psychomotor skills. Practicing on dental simulators helps students master skills in a controlled and safe environment while avoiding patient harm. However, laboratory settings may also present limitations, including frequency of feedback provided by instructors and low ratios of instructors to students. ${ }^{1}$ The clinical setting will complement preclinical courses and reinforce learned concepts by evaluating not only the recall and recognition of specific facts and the mastery of skills, but also students' capacity to apply critical thinking and problem-solving skills and further develop cognitive skills. ${ }^{2}$
The clinical setting is a critical environment for educating new general dentists to become competent, an essential ability to begin independent dental practice. ${ }^{3}$ Traditionally, dental student assessments have included multiple-choice testing, laboratory practicals, daily grades, clinical competency exams, and procedure requirements. ${ }^{2}$ Clinical assessments are meant to test mastery of a competency, which is defined in the American Dental Education Association (ADEA) Competencies for the New General Dentist as "a complex behavior or ability essential for the general dentist to begin independent, unsupervised dental practice. Competence includes knowledge, experience, critical thinking and problem-solving skills, professionalism, ethical values, and technical and procedural skills" (p. 844). ${ }^{3}$ The use of clinical settings, in which students practice for an extended period of time to expand their experience and work consistency, is a valuable method to assess student learning outcomes. ${ }^{2,4}$

Daily clinical grades and clinical assessments including competencies and skills assessments may be used to assess students' proficiency. In a clinical setting, these types of assessments traditionally occur in the presence of a clinical instructor who evaluates 
students based on their interactions with patients and clinical performance. Daily clinical grades are evaluations that occur on a regular basis in which a student, supervised by a clinical instructor, receives a grade for each procedure completed on a patient. In restorative dentistry, students complete either direct or indirect procedures. Direct operative procedures involve preparations and restorations of Class I, II, III, IV, and V lesions. Indirect operative procedures involve preparations and cast or milled restorations. Graded assessments are exams in which a student works independently without the assistance of a clinical instructor and is evaluated on each step of the procedure. For both daily clinical grades and graded assessments, numerical grades are used to appraise students' critical thinking, professionalism, communication and interpersonal skills, health promotion, practice management, and patient care as outlined in the ADEA Competencies for the New General Dentist. ${ }^{3}$

Clinical assessments for dental students can be challenging because while treatment and care for patients are important parts of the learning process, they can add complexity to student-teacher interactions. ${ }^{5}$ Clinical learning and assessment through direct patient care are complicated because the patient is receiving irreversible procedures under the care of a dental student. An instructor must teach the student and prevent harm to the patient. An instructor must also teach the student to respect the patient's needs, demands, values, and expectations. ${ }^{6}$ Tension can result from the learning needs of a student and the duty to prevent harm to the patient. ${ }^{5}$ The clinical teaching setting may interfere with critical feedback to students due to the fact that most discussions between students and covering instructors occur chairside in front of patients. Additionally, assessments may be compromised due to time constraints. Inadequate time will result in students' and teachers' following routines or sequences, doing what is expected without taking advantage of the learning scenario, and not allowing time for students and teachers to engage in collaborative, active learning. ${ }^{7}$ Clinical teaching and assessments can also be impacted by student requirements over patient needs. The practice of using patients for clinical requirements may challenge ethical principles for proper clinical conduct. ${ }^{6}$ Students have many pressures to meet requirements and finish competencies. Dental students have identified requirement-chasing as a major source of anxiety and have expressed concerns about the ethical implications of using patients as educational tools. ${ }^{2}$ Several factors may influence learning and assessment of students in a clinical setting including the uniqueness of the environment, time constraints, and requirement chasing.

An additional factor that may influence assessments is the subjective nature of instructor grading. Evaluations by clinical instructors can be highly subjective and variable. Discrepancies among faculty members may result from unspecified exceptions, no standardized aids for judgment, unspecific methods of observing, unsystematic inspections, differences in background, and other factors. ${ }^{8}$ Implementation of rubrics with levels of achievement and clearly defined criteria is essential. Learning is simplified by use of assessment methods that are consistent and based on meaningful and definite criteria. ${ }^{9}$ Rubrics provide a mechanism by which students can selfassess using the same criteria as faculty, promoting self-awareness and critical thinking in relation to their learning. Rubrics can facilitate effective teaching and refine teaching skills. ${ }^{10}$ In addition, rubrics promote consistency among graders and provide detailed feedback to students.

Faculty calibration is an additional and frequent challenge present in clinical courses. Ideally, a clinical instructor should have participated in preclinical courses before assessing students in the clinical setting since that would allow the instructor to gain calibration while student learning is taking place. When clinical instructors are not assigned to preclinical courses, a lack of standardization occurs, resulting in discrepancies among instructors. Discrepancies can exist in terminology, preparation design, and restoration design. ${ }^{11}$ Faculty calibration is an essential process to allow more consistent evaluation with meaningful feedback to students' performance both preclinically and clinically.

Discrepancies are also present when comparing daily grades and competency/skills assessment exams. Even though it seems logical that daily clinical evaluations would be a true representation of a student's performance on exams, a poor correlation between the two methods has been reported. In a study published in 1983, Berrong et al. reported that daily grades received in an academic year were poorly correlated with performance during competency exams. ${ }^{12}$ An additional finding of that study was that daily grades were positively skewed with minimal distinction between students at the high or low percentile of the cumulative grade point average 
(GPA). Further comparisons of daily clinical grades and clinical/laboratory examinations are warranted. The aim of this study was to compare the daily clinical grades of third-year dental students during routine clinical activities involving direct and indirect operative procedures to clinical and laboratory assessments. We hypothesized that there would be no differences between daily grades and graded clinical assessments. A secondary aim of the study was to compare routine daily clinical grades involving indirect operative procedures to graded laboratory assessments; we hypothesized there would be no differences between daily grades and graded laboratory assessments.

\section{Methods}

This study was approved by the Institutional Review Board of the University of Kentucky (IRB Protocol Number: 16-0170-X2B). The study population was 50 fourth-year dental students enrolled at the University of Kentucky College of Dentistry who had completed the clinical course Restorative Dentistry II (RSD 831) during their third year (2014-15). Study inclusion criteria included completion of all requirements for the clinical course (RSD 831) with final grades submitted. All participants gave informed consent for participation in the study, allowing use of their data for teaching and research purposes.

Variables of interest were daily clinical grades, which involved direct and indirect operative procedures and graded skills assessments (clinical and laboratory assessments). Direct and indirect daily grades and clinical assessments were completed by students under supervision of a calibrated restorative faculty member during the student's clinic session. Faculty members were calibrated with previous exposure to preclinical courses or with faculty development sessions. Laboratory assessments were completed in a dental simulation laboratory under the supervision of a calibrated restorative faculty member who is also a clinical instructor calibrated with previous exposure to preclinical courses.

Direct and indirect daily grades were derived from daily clinical activities in which dental students completed operative procedures on their patients. For these assessments, students were able to seek help from an instructor when needed. Students were graded chairside on the clinical floor by the clinical instructor following completion of the appointment.
Students were evaluated numerically (0-100) on clinical preparedness $(5 \%)$, technical performance (75\%), clinical judgment (15\%), and professionalism (5\%). Each student completed two graded clinical skills assessment exams (clinical assessments) for a Class III composite preparation and restoration and either a Class II amalgam or Class II composite preparation and restoration on a selected patient. These assessments were also graded on the clinical floor by a clinical instructor with evaluation rubrics similar to the direct clinical grades. However, students were expected to work independently without help from the clinical instructor. (The rubrics used for these three assessments are available from the corresponding author.)

For the laboratory assessments, each student completed two bench-top assessments. These assessments consisted of an all-ceramic preparation on an anterior tooth (tooth \#8) and a full metal preparation on a posterior tooth (tooth \#19) performed on a dental simulator. These assessments were de-identified and collectively graded by one calibrated faculty member who also supervises clinical activities. Grading components consisted of four equally weighted sessions ( $25 \%$ each) in which students were evaluated on their technical performance. The laboratory assessment rubrics were exclusively based on technical performance. (A laboratory assessment rubric is available from the corresponding author.) GPA was also considered for analyses to determine possible correlations between GPA and all variables of interest (direct daily grades, indirect daily grades, clinical assessments, and laboratory assessments).

This study had a retrospective design that utilized an existing database. This database was de-identified and converted into three databases for analysis: main database, direct daily grades, and indirect daily grades. The main database included total number of procedures completed by each participant throughout the school year, daily grades for direct and indirect operative procedures, and grades for clinical and laboratory assessments. The direct database included only the number of procedures completed and daily grades for direct operative procedures (direct daily grades) and the grades for clinical assessments. The indirect database included only the number of procedures completed and daily grades for indirect operative procedures (indirect daily grades) and the grades for the laboratory assessments. Individual statistical analyses were conducted in the three databases to determine differences or similarities related 
to the variables of interest. From the direct database, direct daily grades were compared to average grades of clinical assessments. From the indirect database, indirect daily grades were compared to average grades of laboratory assessments. In addition, analyses of the three databases were used to detect relationships among GPA, daily grades (direct and indirect), and clinical and laboratory assessments.

Statistical analyses were conducted using SAS 9.4 (SAS Institute Inc., Cary, NC, USA). The means, t-test, and correlation coefficient procedures were used to complete all analyses. A paired t-test was performed to test the difference between the direct daily grades and clinical assessment grades using the data from the direct database. Another paired t-test was performed to test the difference between the indirect daily grades and laboratory assessment grades using the data from the indirect database. To analyze the relationship between GPA and the variables of interest (daily direct and indirect grades, clinical and laboratory assessments), Pearson's correlation tests were run to check which variables had a strong linear relationship with GPA. Statistical significance was set at a $p$-value of $\leq 0.05$.

\section{Results}

Of the 50 eligible students, one did not agree to participate, so the response rate was $98 \%(\mathrm{~N}=49)$. The study population was 24 to 37 years of age, approximately $47 \%$ female, and predominantly Caucasian (with 5\% Asian, 4\% Hispanic, and 1\% multiracial).

Mean values for the number of completed clinical sessions and all graded assignments (daily grades, clinical assessment grades, and laboratory assessment grades) for the main, direct, and indirect databases are shown in Table 1. For analyses conducted in the direct database, there were no statistically significant differences between direct daily grades (90.4) and clinical assessments (90.6) $(\mathrm{p}=0.2845$; Table 2). For analyses conducted in the indirect database, there was a tendency for lower grades for the laboratory assessments than for the indirect daily grades. There were statistically significance differences between indirect daily grades (90.3) and laboratory assessments (89.6) $(\mathrm{p}=0.0024)$. Pearson's correlation coefficients $(\mathrm{r})$ between GPA and direct daily grades $(\mathrm{p}=0.008)$, indirect daily grades $(\mathrm{p}=0.003)$, and main daily grades $(p=0.001)$ were significant. The Pearson's correla-

Table 1. Distribution of variables for three databases in study

\begin{tabular}{lcc} 
Variable & Database & Mean (Standard Deviation) \\
\hline Number of sessions & Direct & $21.4(4.27)$ \\
& Indirect & $13.0(6.47)$ \\
Daily grades & Main & $34.2(5.67)$ \\
& Direct & $90.4(1.07)$ \\
& Indirect & $90.3(1.28)$ \\
Clinical assessment grades & Main & $90.4(1.03)$ \\
Laboratory assessment grades & Direct \& Main & $90.6(1.47)$ \\
& Indirect \& Main & $89.6(1.35)$
\end{tabular}

Note: Main database included direct and indirect daily grades and average grades of clinical and laboratory assessments. Direct database included direct daily grades and clinical assessment grades. Indirect database included indirect daily grades and laboratory assessment grades.

Table 2. Paired t-tests of daily clinic average grades and assessment grades

\begin{tabular}{lcc} 
Compared Groups & Mean Difference (Confidence Interval) & p-value \\
\hline Direct daily grades and clinical assessment grades & $-0.21(-0.60,0.18)$ & 0.2845 \\
Indirect daily grades and laboratory assessment grades & $0.76(0.28,1.24)$ & $0.0024^{*}$ \\
*Statistically significant difference between two groups & &
\end{tabular}


tions for clinical $(\mathrm{p}=0.06)$ and laboratory $(\mathrm{p}=0.66)$ assessments were not significant. However, there was a weak linear relationship with GPA for all tested variables: daily grades $(r=0.37052)$, indirect daily grades $(\mathrm{r}=0.41604)$, clinical assessments $(\mathrm{r}=0.26355)$, and laboratorial assessments $(\mathrm{r}=0.06366)$.

\section{Discussion}

Analyses of three existing databases were used to test differences and/or similarities between daily clinical evaluations involving direct and indirect operative procedures and two methods of graded skills assessments: clinical and laboratory assessments. Regarding the study's first aim, no statistically significant differences were found between daily clinical grades (direct procedures only) and graded clinical assessments, thus confirming the first hypothesis. On the contrary, regarding the secondary aim, statistically significant differences were found between daily clinical grades (indirect procedures only) and graded laboratory assessments; consequently, the second hypothesis was rejected. We found no statistically significant differences between clinical activities involving daily grades and clinical assessments; however, the analysis revealed statistically significant differences between clinical activities and laboratory assessments.

Contrary to the findings of Berrong et al.'s study, ${ }^{12}$ we found no statistically significant differences between daily clinical grades and clinical assessments. Berrong et al. found a poor correlation between daily grades and practical clinical examinations and concluded that daily grades were positively skewed with minimal distinction between students at high and low ends of the cumulative GPA. Although our study also found a poor correlation between daily clinical grades and GPA and between grades of clinical assessments and GPA, we did not find differences between daily grades and clinical assessments, so it is not possible to infer that clinical grades were positively skewed based exclusively on exams graded in the clinical environment. These discrepancies in findings between the two studies could be explained by methodological differences, making definitive comparisons infeasible. Even though our study had a retrospective design, both daily grades and graded assessments were completed throughout the year, while the practical exams in Berrong et al.'s study were retrospectively analyzed.
Also, Berrong et al.'s study was conducted over 35 years ago, and clinical education has evolved since then.

However, in our study, comparisons of the indirect daily grades in the clinical setting and the laboratory assessments performed on a dental simulator showed that the indirect daily grades were higher than the grades of laboratory assessments, suggesting an inflation of assessments graded in the clinical setting. The fact that students did not score higher in laboratory assessments was unexpected, in that as much as the dental simulator simulates a patient, it will never be the same as working on a human being with all of the physical and psychological variables. The difference might be associated with the two environments in which the assessments took place: the clinical and laboratory settings. The fact that the laboratory assessment procedures were performed on dental simulators while students worked independently from instructors may account to a certain extent for these discrepancies. In the clinical setting, where daily indirect grades are determined, instructors have a duty to prevent harm to the patient. This environment may account for instructor interruption to complete or assist with procedures. Regardless, faculty members must "make a choice to be conscious and deliberate" in their grading activities. ${ }^{13}$ Clinical instructors may be influenced by student effort, resulting in inflation of clinical grades especially for daily clinical grades that are assigned in an environment of generous faculty guidance and feedback to students. On the other hand, laboratory assessments on dental simulators may induce faculty members to be more critical and expect unflawed work.

Another possibility for differences between indirect daily grades and laboratory assessments could be the use of different rubrics for the two assessments. Lower grades for the laboratory assessments could be because the grades were derived solely from technical performance categories. While this might be a possible explanation, it is not likely to be the only factor since indirect daily grades were also heavily graded in technical performance; $75 \%$ of the grade originated from technical performance with the remaining $25 \%$ distributed among the other three criteria (preparedness, judgment, and professionalism).

These findings suggested that the clinical grades, whether daily grades or assessments, might be inflated when compared to laboratory exams. The clinical environment might influence grade inflation. To prevent inconsistencies related to grading, use of standardized rubrics that students and faculty review 
and agree upon before the exams begin is essential. Rubrics establish clear rules for the evaluation, defining and differentiating levels of performance into outstanding, acceptable, or unacceptable. ${ }^{9}$ Additionally, the use of rubrics will give sound feedback to students, document student improvement, and identify areas that need additional emphasis in the curriculum. Standardized rubrics are also likely to minimize problems with faculty calibration. ${ }^{10}$ In an ideal setting, all clinical instructors would have participated in preclinical courses, which is perhaps the most efficient method of faculty calibration. ${ }^{11}$ The reality, however, is that not all clinical faculty members have the opportunity to participate in preclinical courses. There is a constant need to calibrate clinical faculty members, especially the ones not involved in preclinical courses. Faculty development sessions using an audience response system were found effective in calibrating faculty members to ensure consistent instruction for assessing dental student competence. ${ }^{11}$ An additional resource to calibrate faculty would be use of clinical teaching handbooks and clinical manuals, which will familiarize new faculty and part-time faculty with clinical teaching and student assessments. ${ }^{14}$ While faculty development sessions and clinical manuals are important tools, the introduction of systematic rubrics will calibrate not only faculty members but students with terminology, preparation and restoration designs, and specific requirements of an exam. ${ }^{9}$ Overall, development of precise rubrics and faculty calibration are essential in clinical teaching.

Another important aspect in the clinical setting is teaching feedback in the presence of a patient. How can a faculty member teach in the presence of a patient without affecting the student-patient relationship? As described in Irby's review, four key factors distinguish the "excellent" clinical teacher from others: serves as a positive role model, provides effective supervision and mentorship, employs a varied and dynamic approach of teaching, and is a supportive person. ${ }^{15}$ An excellent clinical teacher will employ a dynamic approach of teaching in a patient's presence while providing care and explaining to the student what is happening and why. A clinical teacher will stimulate students' critical thinking by asking questions to assess and plan. This style of teaching reinforces the importance of faculty development sessions in which faculty members are exposed to techniques to improve clinical teaching. Additional roles of the institution to improve clinical teaching are to provide a good mix of patients and clinical experiences, as well as providing a sufficient number of faculty members to allow for consistency in the clinical setting. Students need to be able to work consistently with the same instructors who know their abilities and learning needs. ${ }^{16}$ Implementation of these measures by the institution will also help students to fulfill requirements in a timely manner, minimizing the possibility of requirement chasing.

This study had limitations including its retrospective design and relatively small sample size. Since the study took place at only one dental school, its results may not be generalizable to students at other schools. To minimize bias and confounding variables, a prospective design with larger sample size and multiple schools is warranted. On a positive note, all assessments for this study took place throughout the same year, which would minimize errors due to different levels of skills. The fact that the laboratory assessments were graded by only one instructor is another study limitation. Although the examiner was a clinical instructor calibrated with the same methods as the clinical instructors, this could have influenced the differences between clinical and laboratory assessments. On the other hand, the fact that laboratory assessment grades were done anonymously and collectively may have eliminated bias that might be present in clinical evaluations. Another limitation is the difference between rubrics used for the clinical and laboratory assessments. Further research should focus on a prospective design in which faculty and students would be calibrated and trained with specific rubrics for clinical daily grades and clinical and laboratory assessments. Further research should also focus on more definitive methods of assessments involving the clinical setting to certify appropriate clinical evaluation of dental students with necessary feedback.

\section{Conclusion}

This study found higher grades were given for procedures completed and graded in the clinical setting than assessments completed on a laboratory simulator with blinded grading. This difference was seen for both daily clinical grades associated with daily procedures and graded clinical assessments. Further prospective studies with a larger sample size are needed to explore the possibility of clinical grades being positively skewed. 


\section{Acknowledgments}

The authors would like to acknowledge the Applied Statistics Laboratory at the University of Kentucky. We would also like to thank the following individual for collaboration with data analyses: Kristen McQuerry, PhD, Yan Zhang, and Jin Xie.

\section{REFERENCES}

1. LeBlanc VR, Urbankova A, Hadavi F, Lichtenthal RM. A preliminary study in using virtual reality to train dental students. J Dent Educ 2004;68(3):378-83.

2. Albino JE, Young SK, Neumann LM, et al. Assessing dental students' competence: best practice recommendations in the performance assessment literature and investigation of current practices in predoctoral dental education. J Dent Educ 2008;72(12):1405-35.

3. American Dental Education Association. ADEA competencies for the new general dentist. J Dent Educ 2017;81(7):844-7.

4. Hendricson WD, Kleffner JH. Curricular and instructional implications of competency-based dental education. J Dent Educ 1998;62(2):183-96.

5. Fugill M. Teaching and learning in dental student clinical practice. Eur J Dent Educ 2005;9(3):131-6.

6. Eriksen HM, Bergdahl J, Bergdahl M. A patient-centered approach to teaching and learning in dental student clinical practice. Eur J Dent Educ 2008;12(3):170-5.
7. McMillan W. Making the most of teaching at the chairside. Eur J Dent Educ 2011;15(1):63-8.

8. Taleghani M, Solomon ES, Wathen WF. Non-graded clinical evaluation of dental students in a competency-based education program. J Dent Educ 2004;68(6):644-55.

9. O’Donnell JA, Oakley M, Haney S, et al. Rubrics 101: a primer for rubric development in dental education. J Dent Educ 2011;75(9):1163-75.

10. Brown MC, Conway J, Sorensen TD. Development and implementation of a scoring rubric for aseptic technique. Am J Pharm Educ 2006;70(6):133.

11. Metz MJ, Metz CJ, Durski MT, et al. A training program using an audience response system to calibrate dental faculty members assessing student clinical competence. J Dent Educ 2016;80(9):1109-18.

12. Berrong JM, Buchanan RN, Hendricson WD. Evaluation of practical clinical examinations. J Dent Educ 1983;47(10):656-63.

13. Reybold LE. Faculty ethics: ideal principles with practical applications. J Vet Med Educ 2009;36(4):375-81.

14. Pierangeli L. Developing a clinical teaching handbook and reference manual for part-time clinical faculty. Nurse Educ 2006;31(4):183-5.

15. Irby DM. Teaching and learning in ambulatory care settings: a thematic review of the literature. Acad Med 1995;70(10):898-931.

16. Henzi D, Davis E, Jasinevicius R, Hendricson W. North American dental students' perspectives about their clinical education. J Dent Educ 2006;70(4):361-77. 